\title{
Ecosistema de Manglar de la Bahía de Panamá: Investigaciones en desarrollo
}

\author{
Tejedor-Flores, Nathalia \\ Universidad Tecnológica de Panamá, Centro de Investigaciones Hidráulicas e Hidrotécnicas \\ Sistema Nacional de Investigación (SNI) \\ Panamá, Panamá \\ nathalia.tejedor@utp.ac.pa \\ González, Jaime \\ Universidad Tecnológica de Panamá, Centro de Investigaciones Hidráulicas e Hidrotécnicas \\ Panamá, Panamá \\ jaimegonzalez2@outlook.com \\ Guerra Sosa, Carlos Eduardo \\ Universidad Tecnológica de Panamá, Facultad de Ingeniería Civil \\ Panamá, Panamá \\ guerrasocarlos@protonmail.com \\ Tamayo Martínez, Miguel \\ Universidad Tecnológica de Panamá, Facultad de Ingeniería de Sistemas Computacionales \\ Panamá, Panamá \\ miguelt2296@gmail.com \\ Pinzón, Reinhardt \\ Universidad Tecnológica de Panamá, Centro de Investigaciones Hidráulicas e Hidrotécnicas \\ Sistema Nacional de Investigación (SNI) \\ Panamá, Panamá \\ reinhardt.pinzon@utp.ac.pa
}

\section{Abstract}

The mangrove forests that exist in the world are extremely productive ecosystems, both in the sense of biomass and carbon storage. These mangrove forests store a large amount of carbon, not only at the above ground but also below ground, being in some cases larger carbon sequestration than other similar organisms. Due to this importance, the main objective of this symposium was to present the different research being developed related to the mangrove ecosystem, specifically in the Panama Bay. The speakers presented the 
progress of their research on: the analysis of $\mathrm{CO}_{2}$ fluxes; hydrodynamics of halophytes and the role of vegetation features in the response of the ecosystem to disturbances at the landaquatic interface; analysis of meteorological variables of humidity and air temperature in a mangrove of Juan Diaz; and computer developments in Python for the management of data from the tower in the mangrove of Juan Diaz. All presentations were followed by a question and answer session, focusing on how to implement public policies and binding commitments to better manage and conserve mangrove ecosystems.

Keywords: Mangrove, carbon dioxide fluxes, climate change, meteorological variables, Python.

\section{Resumen}

Los bosques de manglar que existen en el mundo son ecosistemas extremada- mente productivos, tanto en el sentido biomasa como en el almacenamiento de carbono y otros usos. Estos bosques de manglar almacenan gran cantidad de car- bono no solo a nivel aéreo sino por debajo del suelo, siendo en algunos casos mayores secuestradores que otros organismos similares. Debido a esta importancia, el objetivo principal de esta mesa redonda realizada fue presentar las diferentes investigaciones que se están desarrollando relacionadas al ecosistema de manglar, específicamente las investigaciones que actualmente se están desarrollando en la Bahía de Panamá. Los expositores presentaron los avances de sus investigaciones sobre: el análisis de flujos de flujos de $\mathrm{CO}_{2}$; hidrodinámica de las halófitas y el papel de los rasgos de la vegetación en la respuesta del ecosistema a las perturbaciones en la interfaz terrestre-acuática; análisis de las variables meteorológicas de humedad y temperatura del aire en un manglar de Juan Díaz; y desarrollos informáticos en Python para la administración de data proveniente de la torre en el manglar de Juan Díaz. En todas las presentaciones hubo una sesión de preguntas y respuestas, enfocadas sobre cómo implementar políticas públicas y compromisos vinculantes que permitan un mejor manejo y conservación de los ecosistemas de manglar.

Palabras claves: Manglares, flujo de dióxido de carbono, cambio climático, variables meteorológicas, Python. 


\section{INTRODUCCIÓN}

Los bosques de manglar son ecosistemas altamente diversos y productivos en las zonas intertidales de las líneas costeras tropicales y subtropicales [1-2]. Según estimaciones, ocupan el 3\% de la superficie terrestre [3] Su importancia ecológica es inmensa y variada, siendo el hábitat de crianza de moluscos, peces y crustáceos; sirviendo como lugar de encuentro para aves playeras migratorias; previniendo y controlando el flujo de agua hacia y desde la costa y siendo los bosques con mayor densidad de carbono en el trópico, sobre todo en niveles inferiores del suelo [1]

A pesar de que los manglares brindan una serie de importantes servicios ecosistémicos para la humanidad, su existencia está amenazada por la deforestación, el cambio de uso de suelo y el cambio climático [4]. En el 2015, a través de la Fundación Ciudad del Saber y la Coordinación General del Proyecto Parque Urbano de Manglar, se invita al Centro de Investigaciones Hidráulicas e Hidrotécnicas y a la Unidad de Cambio Climático del Ministerio del Ambiente, a conformar el equipo nacional como contraparte de los expertos internacionales, para el desarrollo del componente de investigación sobre monitoreo del flujo de $\mathrm{CO} 2$ y otros parámetros climáticos, asociados a los servicios que presta el manglar; de ahí surge el Proyecto SENACYT de Colaboración Internacional FID16-30 "Análisis de flujos de $\mathrm{CO} 2$ y vapor de agua de un ecosistema de manglar en la Bahía de Panamá", con el objetivo principal de crear metodologías sostenibles estandarizadas y la capacidad institucional que permita a Panamá estimar el intercambio estacional de carbono y agua en zonas de manglares, utilizando métodos estadísticos multivariantes.

El proyecto contempla tres líneas principales de trabajo, tales como la educación ambiental; la participación social, y el desarrollo de la investigación sobre el papel de los manglares en la reducción del riesgo y la adaptación al cambio climático [5], por lo anterior descrito esta mesa redonda realizada durante el Congreso de APANAC tuvo como objetivo principal presentar las diferentes investigaciones que se están desarrollando relacionadas al ecosistema de manglar, específicamente sobre las investigaciones que actualmente se están llevando a cabo en la Bahía de Panamá. Los expositores presentaron los avances de sus investigaciones sobre: el análisis de flujos de flujos de $\mathrm{CO} 2$; hidrodinámica de las halófitas y el papel de los rasgos de la vegetación en la respuesta del ecosistema a las perturbaciones en la interfaz terrestre-acuática; análisis de las variables meteorológicas de humedad y temperatura del aire en un manglar de Juan Díaz; y desarrollos informáticos en Python para la administración de data proveniente de la torre en el manglar de Juan Díaz. El siguiente resumen de mesa redonda tiene la finalidad de recopilar la información presentada por los diversos conferencistas, para lo cual en la Sección 2, se presentan los resúmenes de cada exposición y finalmente en la Sección 3, las conclusiones más relevantes. 


\section{CONFERENCIAS PRESENTADAS}

A continuación, se presenta de manera resumida como fue la presentación el contenido de cada una de las presentaciones:

\section{A. Proyecto FID16-30 "Análisis de flujos de $\mathrm{CO}_{2}$ y vapor de agua de un ecosistema de manglar en la Bahía de Panamá".}

Presentación realizada por la Dra. Nathalia Tejedor, que incluye una breve introducción sobre los ecosistemas de manglar, los objetivos de la investigación, la metodología utilizada (sitio de estudio e instrumentación), resultados obtenidos y conclusiones de los avances realizados en el proyecto. Además, fueron presentadas las publicaciones realizadas por los investigadores del proyecto y estudiantes de tesis: A Bibliographic Review of the Importance of Carbon Dioxide Capture in Mangroves [6], Cuantificación de flujos de $\mathrm{CO}_{2}$ en un ecosistema de manglar en la Bahía de Panamá [7] y A Critical Review on Mathematical Descriptions to Study Flux Processes and Environmental-Related Interactions of Mangroves [8].

B. Hidrodinámica de las halófitas y el papel de los rasgos de la vegetación en la respuesta del ecosistema a las perturbaciones en la interfaz terrestre-acuática

Presentación realizada por el Ing. Jaime González, quien hizo referencia a los aspectos relevantes del proyecto "Exploring the influence of above- and below-ground plant trait plasticity and its parameterization in vegetation hydrodynamics modules within land surface models". Inició su presentación con una breve introducción sobre el ecosistema de manglar y a su comportamiento especial por crecer en zonas intermareales, por lo que rara vez están limitados por la disponibilidad de humedad en la zona de las raíces. Explicó que, durante la estación seca, estos ecosistemas han demostrado se comportan de forma más parecida a los ecosistemas semiáridos que a los bosques húmedos. El proceso de exclusión de la sal de las aguas marinas y salobres durante la absorción del agua de las raíces proporciona una energía adicional en el continuo suelo-planta-atmósfera. Esta adaptación es responsable de las altas tensiones del xilema que dan lugar al cierre estomático y a la reducción de la transpiración, las reducciones de la transpiración provocan un aumento del flujo de calor sensible en lugar del calor latente, lo que conduce a un balance energético característico de un sistema con limitación de agua. Finamente el Ing. González expuso la metodología utilizada para estudiar este fenómeno en la Bahía de Panamá. Figura 1. 


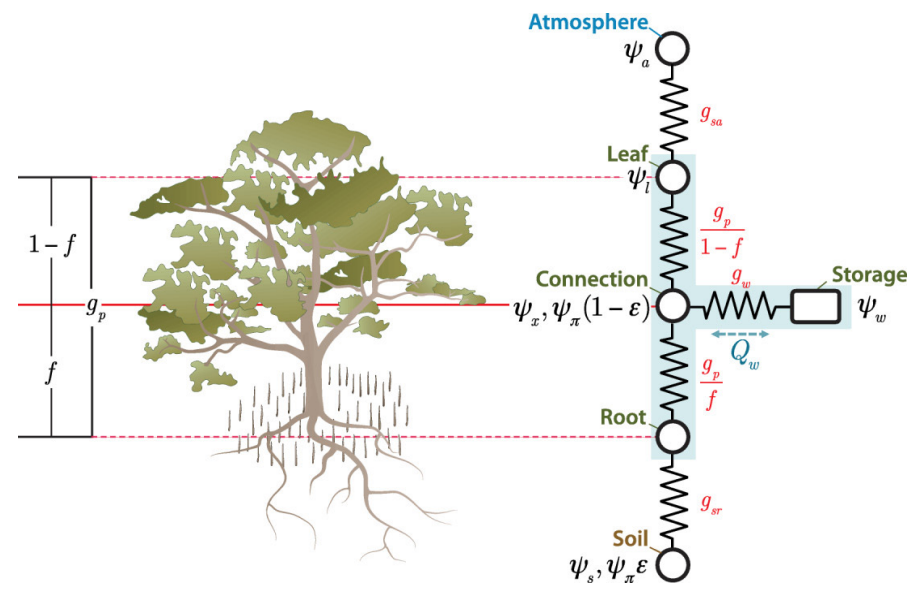

Figura 1. Representación conceptual del flujo de savia con dependencia de salinidad del suelo. Fuente: [9].

C. Análisis de las variables meteorológicas de humedad y temperatura del aire en un manglar de Juan Díaz

Presentación realizada por el Ing. Carlos Guerra, quién explico que en el marco del Proyecto FID16-30, se desarrolló su tesis de licenciatura en ingeniería ambiental, titulada "Análisis de las variables meteorológicas de humedad y temperatura del aire en un manglar de Juan Díaz", con el objetivo de analizar el gradiente de temperatura en un ecosistema de manglar. La investigación fue realizada con datos de los diez sensores de temperatura del aire y los dos sensores de humedad relativa colectados durante los años 2017 y 2018. Figura 2.

D. Desarrollos informáticos en Python para la administración de data proveniente de la torre en el manglar de Juan Díaz

En el marco del Proyecto FID16-30, el estudiante Miguel Tamayo, desarrolla la tesis de licenciatura en Ingeniería de Sistemas y Computación, titulada "Desarrollos informáticos en Python para la administración de data proveniente de la torre en el manglar de Juan Díaz". En este proyecto de investigación se obtendrán datos de los sensores del manglar de la Bahía de Panamá. Estos datos requieren de un análisis muy detallado y preciso, por lo que un software óptimo y potente es un requerimiento fundamental para el desarrollo del proyecto. Este software nos permitirá obtener de forma gráficas los datos obtenidos de los sensores y mostrarlo de manera comprensible para que el investigador tenga una mejor visión y entendimiento con lo ocurrido en cuanto al flujo de $\mathrm{CO}_{2}$ y de vapor de agua, permitiéndole obtener conclusiones gracias a la simplicidad con la que los datos se mostrarán. 


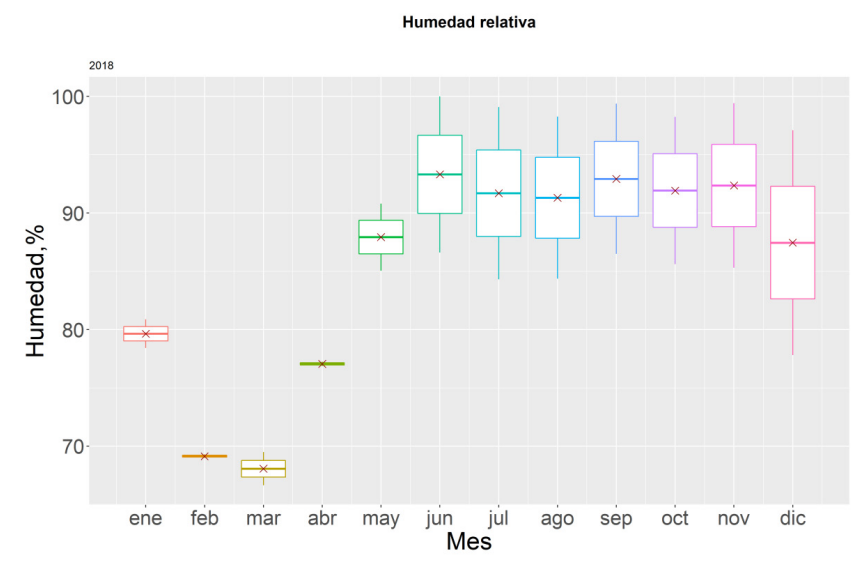

Figura 2. Boxplot anual para serie de humedades mensuales del 2018. Fuente: Carlos Guerra.

\section{CONCLUSIONES}

Con las investigaciones presentadas y los estudios relacionados de los flujos de carbono, se demuestran los beneficios de la conservación de los manglares y las mejoras que pueden aportar al ecosistema o a la temática del cambio climático. Los análisis de los datos presentados indican que el sistema de adquisición de data y condiciones del ecosistema funciona correctamente. El proyecto principal (FID16-30), todavía se debe evaluar otros datos y corregir algunos factores que no sean los adecuados.

\section{Referencias}

[1] Bouillon, S., Borges, A. V., Castañeda-Moya, E., Diele, K., Dittmar, T., Duke, N.C., Kristensen, E., Lee, S.Y., Marchand, C., Middelburg, J.J., Rivera-Monroy, V.H., Smith, T.J. Y Twilley, R.R., "Mangrove production and carbon sinks: A revision of global budget estimates. Global Biogeochemical Cycles", vol. 22, no. 2. ISSN 08866236, 2008.

[2] Spalding, M., Kainuma, M. Y Lorna, C., "Atlas Mundial de los Manglares", 2.a ed. Londres, Okinawa: Organización Internacional de las Maderas Tropicales (OIMT); Sociedad Internacional para los Ecosistemas de Manglares (SIEM). ISBN 978-4-906584-15-4, 2011.

[3] Autoridad Nacional del Ambiente y Autoridad de los Recursos Acuáticos de Panamá," Manglares de Panamá: importancia, mejores prácticas y regulaciones vigentes" [en línea]. 1.a ed. Ciudad de Panamá, República de Panamá: Editora Novo, S.A. ISBN 978-9962651864, 2013.

[4] R. Diana, V. B. Arifanti, D. Hadriyanto, J. B. Kauffman, and D. Murdiyarso, "Carbon dynamics and land use carbon footprints in mangrove-converted aquaculture: The case of the Mahakam Delta, Indonesia," For. Ecol. Manage., vol. 432, no. August 2018, pp. 17-29, 2018.

[5] Centro de Investigaciones Hidráulicas e Hidrotécnicas, "Análisis de Flujo de $\mathrm{CO}_{2}$ y Vapor de Agua de un Ecosistema de Manglar", [en línea]. [Consulta: 21 febrero 2019]. Disponible en: http://manglarcarbono.utp.ac.pa/, 2019.

[6] J. González, N. Tejedor-Flores and R. Pinzón, “A Bibliographic Review of the Importance of Carbon 
Dioxide Capture in Mangroves," 2019 7th International Engineering, Sciences and Technology Conference (IESTEC), 2019, pp. 126-131, doi: 10.1109/IESTEC46403.2019.00-89.

[7] Jaime González, Nathalia Tejedor-Flores, Reinhardt Pinzón, Ana Franco, "Cuantificación de flujos de $\mathrm{CO}^{2}$ en un ecosistema de manglar en la Bahía de Panamá", Políticas Públicas Territoriales y Desarrollo Regional, pp. 243- 255, Editorial Thompson Reuters-Aranzadi, 2020.

[8] J. Brooks, M. Chen Austin, D. Mora, and N. Tejedor-Flores, "A Critical Review on Mathematical Descriptions to Study Flux Processes and Environmental-Related Interactions of Mangroves," Sustainability, vol. 13, no. 12, p. 6970, Jun. 2021.

[9] Perri, S., D. Entekhabi, and A. Molini, "Plant osmoregulation as an emergent watersaving adaptation", Water Resour. Res., 2018.

\section{Autorización y Licencia CC}

Los autores autorizan a APANAC XVIII a publicar el artículo en las actas de la conferencia en Acceso Abierto (Open Access) en diversos formatos digitales (PDF, HTML, EPUB) e integrarlos en diversas plataformas online como repositorios y bases de datos bajo la licencia CC:

Attribution-NonCommercial-ShareAlike 4.0 International (CC BY-NC-SA 4.0) https://creativecommons. org/licenses/by-nc-sa/4.0/. Ni APANAC XVIII ni los editores son responsables ni del contenido ni de las implicaciones de lo expresado en el artículo. 